\title{
Investigation of activated aluminum as a component of cellular concrete with complex porosation
}

\author{
Viktoria Nelyubova ${ }^{1 *}$ and Artem Sumin $^{2}$ \\ ${ }^{1}$ BSTU named after V. G. Shukhov, Department of Materials Science and Technology, 308012 \\ Belgorod, Russia \\ ${ }^{2}$ LefargeHolcim Russia, 109028 Moscow, Russia
}

\begin{abstract}
In this paper indicators of the quality of activated aluminum, proposed for use as a poreforming agent in the production of cellular concrete with complex porisation are presented. The efficiency of ultrasonic homogenization of the poreforning agent in the presence of a mineral modifier of cellular concrete which provides high stability of the suspension in time is substantiated.
\end{abstract}

\section{Introduction}

Non-autoclaved hardening cellular concrete is one of the leaders of modern effective heatinsulating materials. At the same time, the use of complex mixture porisation allows to balance the shortcomings of foam and gas technologies, ensuring the production of a cellular composite of a given high quality. Traditionally, in the production of aerated concrete, an aluminum paste is used as gas-forming agent. Paste is a mixture of aluminum powder and surface-active agent of high concentration. However, it is known that the use of such pastes together with foaming agents is practically impossible, since the paste shell adversely affects the foaming processes. In addition, these gassing agents have an "explosive" character of gas release: a large volume of gas in a short time. In the works performed in BSTU named after V.G. Shukhov [1-3] the possibility of using an unconventional nanodispersed gassing agent (NDG) for obtaining low-density heatinsulating foam gas concrete, the use of which excludes the stated drawbacks of aluminum powder and allows to obtain products of required quality.

In this paper, the use of activated aluminum AA-T/7 is proposed. To substantiate the possibility of its use as a gas-forming agent, the granulometric composition, the kinetics of gas release, the stability of the aqueous suspension, and also the microstructure were studied. A comparison of the obtained data was made with the characteristics of an aluminum paste and a nanodisperse gassing agent.

\footnotetext{
* Corresponding author: nelubova@list.ru
} 


\section{Materials and methods}

Aluminum paste STAPA Alupor manufactured by limited liability company "Eckart". (Table 1), nanodispersed (Table 2) and activated aluminum AA-T/7 produced by limited liability company "Ecoenergotech" obtained on the basis of ASD-4 aluminum powder (Table 3).

Table 1. Characteristics of aluminium paste STAPA Alupor

\begin{tabular}{|l|c|}
\hline \multicolumn{1}{|c|}{ Characteristics } & Standard value \\
\hline Rest on a sieve $45 \mu \mathrm{m}, \%$, not more than & 20 \\
\hline Particle size, $\mu \mathrm{m}$ & 35 \\
\hline Volatile-matter content, $\%$ & $70 \pm 2$ \\
\hline Acive aluminium content, \%, not less than & 90 \\
\hline $\begin{array}{l}\text { Gas evolution at } 25^{\circ} \mathrm{C} \text { after: } \\
10 \text { min, } \mathrm{ml}, \text { not less than } \\
16 \text { min, } \mathrm{ml}, \text { not less than }\end{array}$ & 60 \\
\hline
\end{tabular}

Table 2. Characteristics of nanodispersed gas-forming agent

\begin{tabular}{|l|c|}
\hline \multicolumn{1}{|c|}{ Characteristics } & Standard value \\
\hline Mean median particle size, $\mu \mathrm{m}$ & 10 \\
\hline Content: & 80 \\
Al, \% not less than & 20 \\
Polyethyleneglycol, \% not more than & \\
\hline
\end{tabular}

Table 3. Properties of the aluminum powder used to prepare AA-T/7

\begin{tabular}{|l|c|}
\hline \multicolumn{1}{|c|}{ Characteristics } & Standard value \\
\hline Powder grade & АСД-4 \\
\hline Bulk density, $\mathrm{g} / \mathrm{cm}^{3}$, not less than & 0,96 \\
\hline Acive aluminium content, \%, not less than & 99 \\
\hline Humidity, $\%$, not more than & 0,2 \\
\hline Specific surface, $\mathrm{m}^{2} / \mathrm{kg}$ & $90-150$ \\
\hline
\end{tabular}


The total particle size distribution of the gassing agents was evaluated using an Analysette 22 NanoTec plus laser particle size analyzer. Granulometric composition of the finely dispersed fraction using the DelsaNano particle size and zeta potential detector in the shared knowledge center "Arktika" at N(A)FU named after M.V. Lomonosov. For this purpose, the colloidal fraction was separated as follows: all the gas-forming agents were pre-mixed with distilled water until a translucent suspension was obtained. Further, the resulting aqueous suspensions were set to rest until a coarse fraction sedimented. The resulting solution with particles suspended in the volume came to the study.

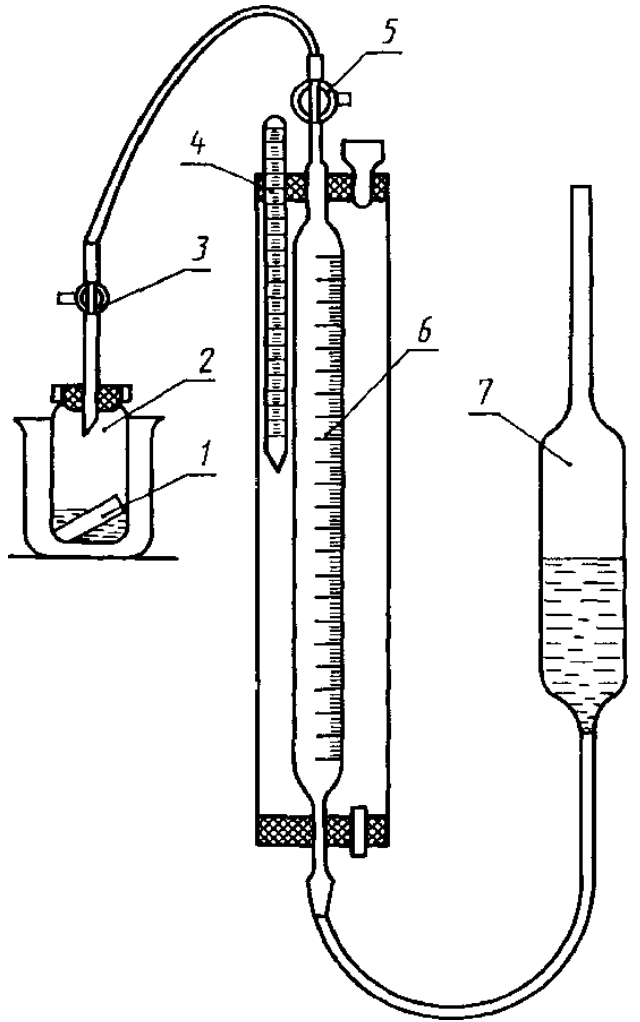

Fig. 1.1 - glass vial; 2 - vessel;

3,5 - connecting cock; 4 - thermometer;

6 - burette 7 - glass vessel
The gas-forming ability and kinetics of gas release of aluminum agents were studied in an alkaline medium using a gasvolume meter (Fig. 1). To do this, a sample of a gas-forming agent weighing $0.1 \mathrm{~g}$ is placed in a test tube which is slanted in the reaction vessel. $30 \mathrm{ml}$ of a sodium hydroxide solution with a mass fraction of $20 \%$ are poured into the reaction vessel. The reaction vessel is tightly closed with a lid and shaken to transfer the gassing agent from the tube into the solution. The measurements are carried out until the gassing process is completely stopped. For the purity of the experiment, the experiment is repeated 2-3 times.

The structure of suspensions of gassing agents obtained by different methods was studied using a POLAM P-312 polarization microscope.

The microstructure of the materials was studied using a high-resolution scanning electron microscope TESCAN MIRA 3 LMU. The samples were studied under high vacuum conditions.

To distribute the gassing agent in an aqueous medium, ultrasonic disperser UZD1-1.6/22 with a constant operating frequency of $24 \mathrm{kHz}$ was used.

\section{Results and discussions}

Analysis of the granulometry of aluminum components indicates a greater dispersion of activated aluminum AA-T/7 and NDG compared to aluminum paste. At the same time the dispersity of NDG and AA-T/7 is comparable. Unconventional blowing agents in comparison with aluminum paste differ with polydisperse composition (Fig. 2). The granulometry of a classical gassing agent is represented by a peak of particle sizes in the region of $100 \mu \mathrm{m}$. The nature of AA-T/7 granulometry is comparable to that of NDG: both microdispersed and finely dispersed fractions are present in the composition.

Activated aluminum, offered for use in research is characterized by peaks in the regions $2-5,10-20,50-70 \mu \mathrm{m}$. At the same time a fraction with a particle size of less than $0.1 \mu \mathrm{m}$ is contained only in non-traditional gassing agents. However, the content of this fraction in the nanodispersed gassing agent is twice as high as in the case of activated aluminum. 


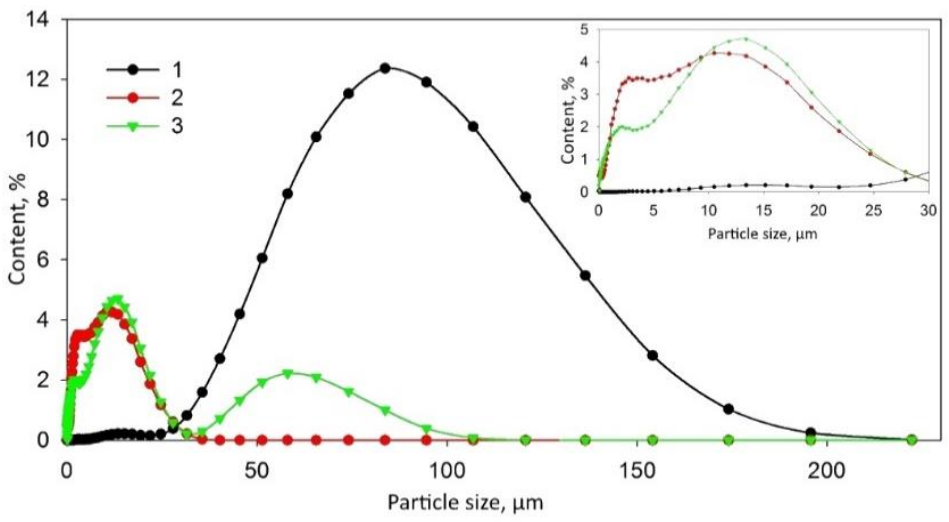

Fig. 2. Granulometric composition of the gas-forming agents:

1 - aluminum paste; 2 - nanodispersed gas-forming agent; 3 - activated aluminum

The colloid fraction of gas-forming agents is substantially different in dispersion. The intermediate position is occupied by activated aluminum with particle sizes in the range $0.1-0.6 \mu \mathrm{m}$ with a peak at a particle size of $0.2 \mu \mathrm{m}$. Such small particle size of the colloid fraction of gas generators will allow to obtain gas bubbles of various micron size, which will lead to the formation of a fine-cellular porous structure of the composite.

Analysis of the kinetics of gas release shows the similarity of the nature of the process in case of aluminum paste and activated aluminum: an intensive increase in the amount of gas is observed in a short time (Fig. 3). In the case of paste, the maximum reaction rate is noted up to 40 seconds, then the rate of gas release decreases and remains practically unchanged until the end of the reaction. Activated aluminum is characterized by a higher reaction rate and a greater volume of gas formed in its result. In this case, the total volume of gas formed as a result of the reaction of the active substance and alkali in the case of NDG and AA-T/7 is 1,8 times higher than that for the aluminum paste. This is due to the granulometry of the gas-forming agents. As was shown earlier, the aluminum paste is characterized by a monodisperse composition with a micron particle size. In this connection, the reaction of the active substance and alkali occurs rapidly enough with the release of a significant amount of gas.

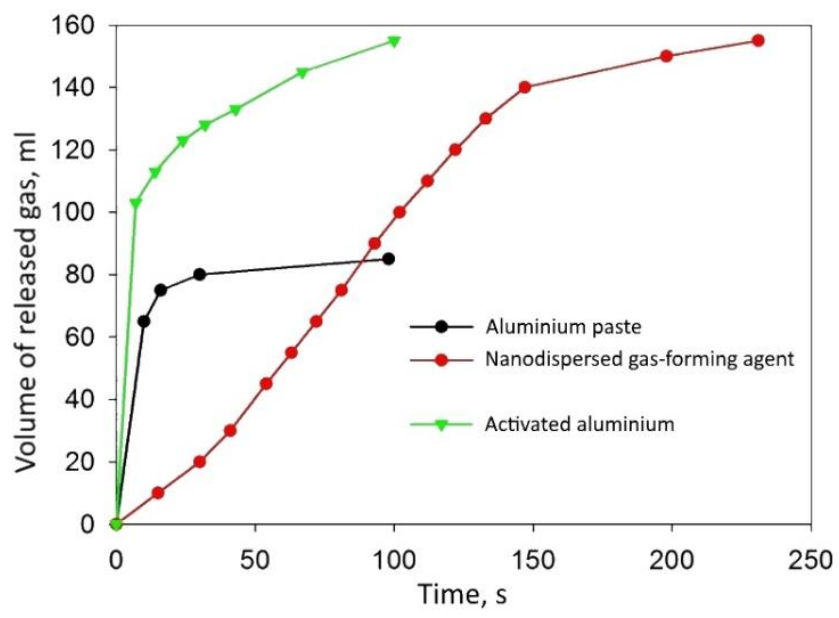

Fig. 3. Cinetics of gas release for studied gas-forming agents 
Activated aluminum occupies an intermediate position. The granulometric composition of the solid substance of this agent is represented by microparticles and nanofraction. This facilitates, on the one hand, the rapid reaction of aluminum and alkali, and on the other, the formation of a larger volume of gas during the interaction. Thus, the short time of gas release of activated aluminum in conjunction with the large volume of gas formed as a result of the reaction, when used as a gasifier, will contribute to the intensification of the formation of porous structure of foamgasconcrete and will allow the creation of a heteroporous structure of the composite.

In connection with the similarity of the results of the studies for activated aluminum and a nanodispersed gas-forming agent, the latter was excluded as a reference sample for further experiments.

The properties of gas-forming agents will be affected not only by their granulometry, i.e. particle size, but also their shape. Thus, the aluminum paste is characterized by a scaly form of particles (Fig. 4, a), whose width reaches $60 \mu \mathrm{m}$. In the general structure of the material, "packages" of particles predominate, the thickness of which is about $0.1 \mu \mathrm{m}$. Activated aluminum is represented by aggregates of spherical particles (Fig. 4, b). "Spheres" AA-T/7 differ with significant polydispersity - from $10 \mathrm{~nm}$ to 0.3 microns. Some particles are characterized by a large size that is within 1-2 $\mu \mathrm{m}$. It is obvious that the shape and size of the particles, regardless of the type of gas-forming agent, will have a significant effect on the distribution of gassing agents in the molding mixture.
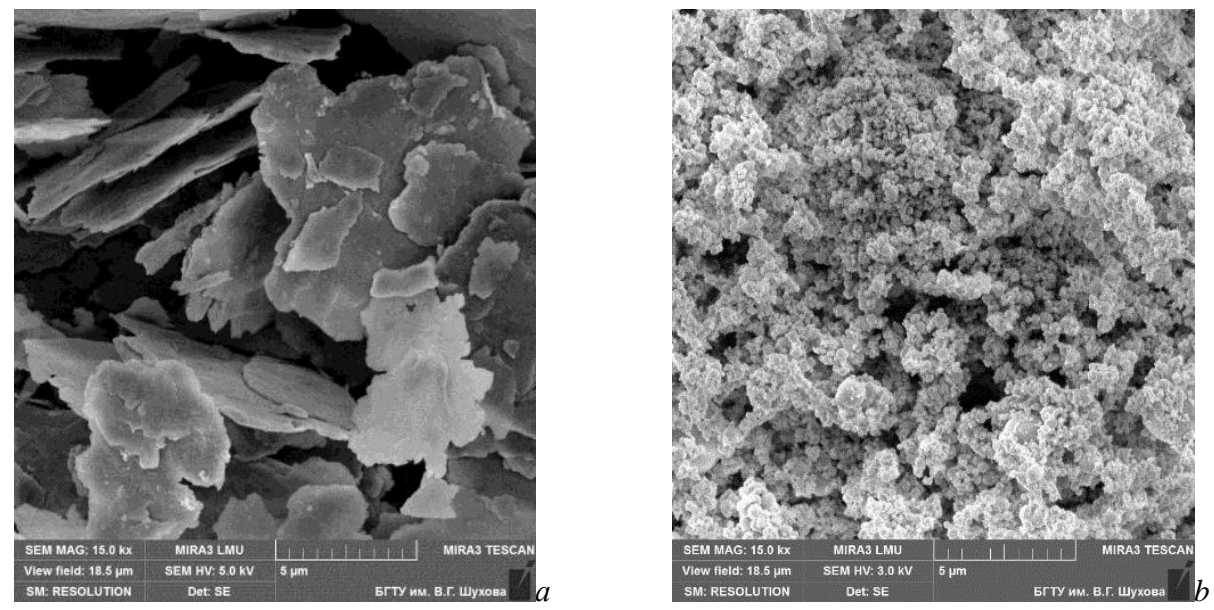

Fig. 4. Microstructure of gas-forming agents: $a$-aluminium paste; $b$-activated aluminium

It is known that for the qualitative distribution and, as a consequence, to ensure uniform gas formation throughout the mixture, the blowing agents are introduced into the concrete mixtures in the form of an aqueous suspension. In this case, the premixing of the components, as a rule, takes place by means of usual mechanical mixing. In this connection, the effect of the method for obtaining a suspension of a gas-forming agent on the quality of the distribution of a gassing agent in an aqueous medium is studied. The mechanical mixing of aluminum components is significantly different in the process time. So, in the case of aluminum paste, a stable homogenized system is achieved 70 seconds from the start of mixing (with equal volumes of capacity). While the use of activated aluminum to obtain a slurry reduces the mixing time by 1.75 times (40 seconds). The explanation of this fact is the shape of the particles of the gas-forming agents.

The structure of the aluminum paste is represented by layered particle packets, so a large amount of time is required to uniformly distribute them throughout the volume of the 
aqueous dispersion medium, which is associated with the need to destroy the laminated package. Activated aluminum is easier to homogenize due to the spherical shape of the particles. In this case, the particles have a minimum number of points of contact between themselves, and, consequently, they can be separated from each other without much effort. In addition, spheres are easier "lubricated" by the dispersion medium (water), which leads to their uniform distribution in less time, the so-called bearing effect works.

A number of authors have proved the effectiveness of using ultrasound for the qualitative distribution of disperse components in the volume of a dispersive medium of various compositions [4-5]. In this connection, an attempt was made to obtain a stable aluminum suspension using an ultrasonic device.

According to the data obtained, the application of ultrasonic action promotes the qualitative distribution of gas-forming agents in the aquatic environment irrespective of their type. The time for obtaining the suspension is reduced to 10 seconds, which is 4 times less than in the case of obtaining a suspension by mechanical stirring for activated aluminum, and 7 times for aluminum paste. In this case, in the case of activated aluminum, a certain "inhibition" of the process is noted, but by the fifth second of the exposure, a uniform distribution of the component over the volume of the dispersion medium is visually observed. The use of ultrasound to mix the aluminum paste leads to filling with its particles of the entire volume of water after already 2 seconds. Nevertheless, complete homogenization of this mixture is visually indicated only to 10 seconds, which is twice the time of obtaining a suspension based on activated aluminum.

These assumptions about the quality of the particle distribution are confirmed by an analysis of the microstructure of aluminium suspensions obtained by means of an ultrasonic device. In the case of aluminium paste, the size of individual clusters consisting of aggregates of solid particles is reduced to 2-3 microns in comparison with the suspension obtained by mechanical mixing (Fig. 5a). The use of ultrasound to homogenize activated aluminum promotes the peptization of the suspension. As a result, a system is formed consisting of individual particles or aggregations of small size separated by a thin layer of the aqueous medium (Fig. 5, b).

Previously performed studies proved the effectiveness of using a nanostructured modifier in the production of cellular concrete of various hardening types [6-7]. It can be assumed that obtaining a suspension of activated aluminum in the presence of a nanostructured modifier will help to stabilize the gas-forming agent in the volume of the aqueous medium. To confirm this thesis, the features of the formation and structure of the system "activated aluminum-nanostructured modifier-water" were studied. This suspension was obtained by means of an ultrasonic device.
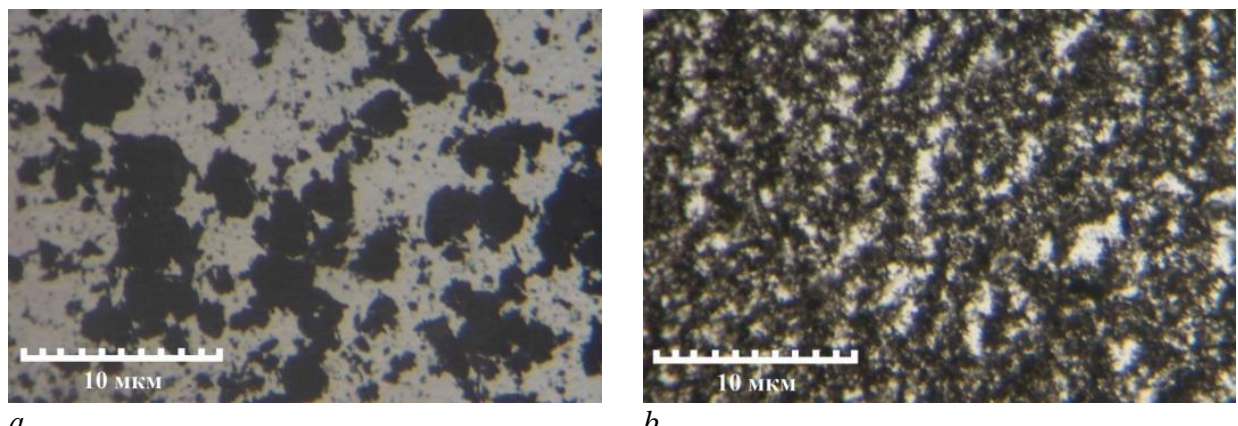
$b$

Fig. 5. Microstructure of suspensions after ultrasonic treatment:

$a$ - aluminum paste; $b$ - activated aluminum 
The process of obtaining a suspension can be divided into several stages. In the first seconds, the finely dispersed fraction of the nanostructured modifier is actively distributed over the volume of the dispersion medium. Further, the volume is filled between the particles of NM with activated aluminum. At the last stage, the microdispersed component of the modifier is involved in motion. All this in combination after 10 seconds of mixing leads to the formation of a uniform stable system.

The microstructure of the homogenized suspension is characterized by a uniform distribution of the particles of the aluminum gas-forming agent in the "water-NM" medium (Fig. 6, a). Clustering of the aluminum component is practically not observed. Each individual particle is surrounded by a shell of a dispersion medium consisting of water and finely divided components of the modifier.

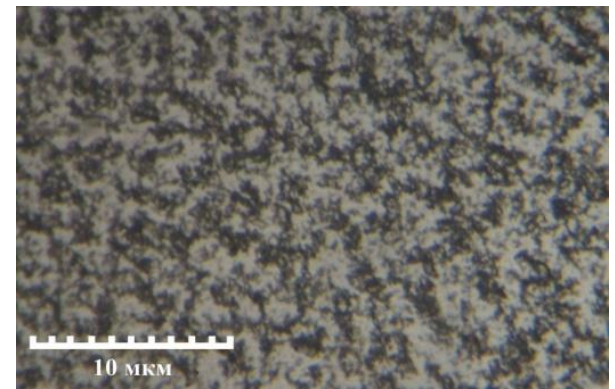

$a$

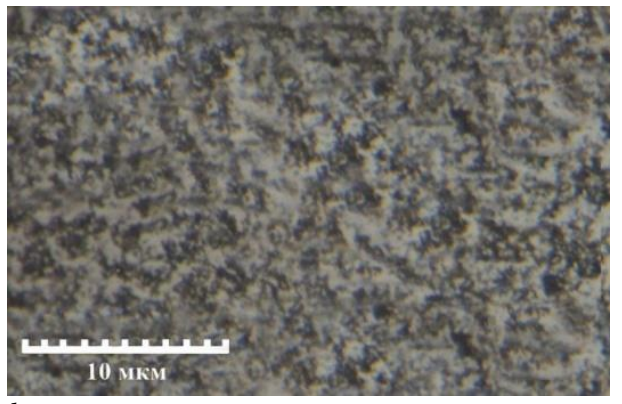

$b$

Fig. 6. Microstructure of the "AA-T/7-NM-water" suspension after ultrasonic mixing: $a$ - freshly prepared; $b$ - after repeated mechanical mixing

To substantiate the effectiveness of the proposed technical solution, the work studied the stability ("survivability") of the suspension in time. It was found that the complete separation of the dispersion medium and the dispersed phase occurs after 12 hours. Herewith the separation of the dispersed phase into activated aluminum and the nanostructured modifier does not occur.

Mechanical mixing of this system after its separation leads to homogenization of the mixture to its initial state: no visual differences are observed. This fact is confirmed by the microstructure of the suspension "AA-T/7-NM-water" studied using a polarization microscope: complete peptization of solid particles (activated aluminum and a nanostructured modifier) in an aqueous medium is noted (Fig. 6, b). Therefore, despite the separation of the system over time, no additional ultrasonic action is required to uniformly distribute particles over the volume of the aqueous medium. It is necessary and sufficient to mechanically briefly mix the components, that leads to the return of the suspension to its original state.

\section{Conclusion}

Thus, the possibility of using activated aluminum as a gas-forming agent in the production of non-autoclaved cellular composites has been demonstrated. The polydisperse composition of the gas-forming agent will contribute to the formation of the gas volume necessary for porosity in a shorter time. The spherical shape of the gas-forming agent particles will optimize the distribution of the component in the aqueous medium. The efficiency of homogenization of activated aluminum in the presence of a nanostructured modifier has been proved to be due to ultrasonic action, which leads to an intensification of 
the processes of distribution of different dispersed components of the modifier and gasforming agent, and also to the stability of the system (no sedimentation).

The work was financially supported with the President's Grant for Young Candidates MK-5980.2018.8 and within the framework of the implementation of the Program for the Development of a Flagship University on the basis of the BSTU named after V.G. Shukhov.

\section{References}

1. V.V. Strokova, A.B. Bukhalo, Building Materials (2008)

2. V.V. Strokova, A.B. Bukhalo, Building Materials (2001)

3. A.V. Sumin, V.V. Strokova, V.V. Nelubova, S.A. Eremenko Building Materials (2016)

4. E.V. Korolev, A.S. Inosemtsev, Building Materials (2012)

5. E.V. Korolev, M.I. Kuvshinnikova, Building Materials (2010)

6. V. Nelubova, V. Strokova, A. Cherevatova, N. Altynnik, K. Sobolev, Materials Research Society Symposium Proceedings (2014)

7. V. Nelyubova, N. Pavlenko, D. Netsvet, IOP Conference Series: Materials Science and Engineering (2015) 